\title{
O uso da técnica de micromarsupialização modificada no tratamento de rânula
}

\section{bilateral: Relato de caso clínico}

The use of the modified micromarsupialization technique in the treatment of bilateral ranula: A clinical case report

El uso de la técnica de micromarsupialización modificada en el tratamiento de ránula bilateral: Reporte de caso clínico

Abrian Andre Gonzalez

ORCID: https://orcid.org/0000-0002-4760-5635

Universidad de Monterrey, México

E-mail: abrianglz@gmail.com

Amanda Silva Nascimento

ORCID: https://orcid.org/0000-0001-7493-4736

Universidade Federal de Pernambuco, Brasil

E-mail: amandasilva_n@hotmail.com

Ana Cléa Aguiar de Araújo Lima

ORCID: https://orcid.org/0000-0002-9988-7988

Centro Universitário de João Pessoa, Brasil

E-mail: anaclea.odontologia@gmail.com

Carlos Augusto Pereira do Lago

ORCID: https://orcid.org/0000-0001-9457-714X

Universidade de Pernambuco, Brasil

E-mail: carloslago1810@hotmail.com

Felipe Ricardo Cisneiros Brito

ORCID: https://orcid.org/0000-0001-8222-9033

Universidade de Pernambuco, Brasil

E-mail: felipe.cisneiros@upe.br

Greiciane Miguel de Azevedo Santos ORCID: https://orcid.org/0000-0001-7876-3655

Universidade de Pernambuco, Brasil

E-mail: greiciane_azevedo@outlook.com

João Victor Souza Pinto

ORCID: https://orcid.org/0000-0003-4224-3492

Centro Universitário de João Pessoa, Brasil

E-mail: jvsp.odontologia@gmail.com

Leandro Pimentel Cabral

ORCID: https://orcid.org/0000-0002-4094-1345

Universidade Federal de Pernambuco, Brasil

E-mail: cabral.leop@gmail.com

Nilton José da Silva Filho

ORCID: https://orcid.org/0000-0001-5802-8362

Universidade Federal de Pernambuco, Brasil

E-mail: niltonfjh@gmail.com

Priscilla Sarmento Pinto

ORCID: https://orcid.org/0000-0002-2376-4383

Universidade Federal de Pernambuco, Brasil

E-mail: priscillasarmentop@gmail.com

Ronaldo Gabriel Martiniano da Silva

ORCID: https://orcid.org/0000-0001-9248-9277

Centro Universitário Tiradentes de Pernambuco, Brasil

E-mail: ronaldo.rgms77@gmail.com

Walter Ferreira Marinho Neto

ORCID: https://orcid.org/0000-0002-3088-2302

Centro Universitário Brasileiro, Brasil

E-mail: walter.fmn@gmail.com 


\title{
Resumo
}

Os fenômenos de extravasamento de muco resultam de um trauma mecânico ao ducto excretor de uma glândula salivar, levando ao acúmulo de mucina no tecido conjuntivo subjacente. São classificados em mucocele e rânula, a depender de sua localização. O tratamento é cirúrgico, variando da marsupialização, remoção definitiva de lesão, além de outras técnicas de descompressão. Relatar um caso clínico de rânula bilateral tratada através da técnica da micromarsupialização modificada com fio de algodão 0. Paciente do sexo feminino, 84 anos, leucoderma e hipertensa deu entrada no serviço de urgência/emergência do Hospital da Restauração Governador Paulo Guerra - Recife - Pernambuco - Brasil apresentando aumento de volume assintomático bilateral em região de assoalho bucal e discreta disfagia. De acordo com o conjunto de avaliações clínicas (anamnese e exames físicos gerais e loco-regionais) e complementares, obteve-se como diagnóstico uma lesão patológica advinda do extravasamento de muco para os tecidos adjacentes denominada rânula, no qual apresentou uma resposta clínica satisfatória ao tratamento de micromarsupialização modificada com fio de algodão número 0 . A técnica da micromarsupialização modificada no tratamento de rânula mostrou sucesso do tratamento, e possui a vantagem de ser um procedimento de simples realização, tempo operatório reduzido, pouco invasivo e com menor risco de danos a estruturas anatômicas.

Palavras-chave: Rânula; Glândula Sublingual; Patologia bucal.

\begin{abstract}
The phenomena of mucus extraversion results from a (mechanical) trauma of the excretory ducts of a salivary gland, which leads to an accumulation of mucin in the underlying connective tissue of the ducts. These results are classified as mucoceles and ranulas depending on their location. Treatment is surgical, ranging from marsupialization to permanent lesion removal in addition to other decompression techniques. The objective of this article is to report a clinical case in which a bilateral ranula was treated using the modified micromarsupialization technique with a cotton thread suture in size 0 . Female patient with 84 years of age presenting leucoderma and hypertension was admitted into the Emergency Room of the Hospital da Restauração Governador Paulo Guerra in Recife, Pernambuco, Brazil, presenting an asymptomatic bilateral volume increase in the oral floor region and mild dysphagia. According to the clinical assessments (anamnesis, general and loco-regional physical examinations) and complementary evaluations, a pathological lesion resulting from the leakage of mucus into the adjacent tissues called a ranula was obtained as a diagnosis in which it presented a satisfactory clinical response with the micromarsupialization treatment modified with cotton thread suture size 0 . The modified micromarsupialization technique for the treatment of a granula showed success and possess the advantage of being a simple procedure reducing operative times. This procedure is also minimally invasive and shows less risk of damage to the surrounding anatomical structures.
\end{abstract}

Keywords: Ranula; Sublingual gland; Oral pathology.

\section{Resumen}

Los fenómenos del Extravasación de mucos son el resultado de un trauma mecánico al conducto excretor de una glándula salival, lo que conduce a la acumulación de mucina en el tejido conectivo subyacente. El tratamiento es quirúrgico, que va desde la marsupialización, la eliminación definitiva de lesiones y otras técnicas de descompresión. Informar de un caso clínico de rugby bilateral tratado utilizando la técnica de micromarsupalización modificada con hilo de algodón 0 . Pacientes del sexo femenino, 84 años, leucoderma y hipertensa fue ingresada en el departamento de urgencias/emergéncias del Hospital da Restauración Paulo Guerra - Recife - Pernambuco - Brasil presentando un aumento en el volumen asintomático bilateral en la región del piso oral y disfagia leve. De acuerdo con el conjunto de evaluaciones clínicas (anamnesis y exámenes físicos generales y locorregionales) y complementarios, se obtuvo como diagnóstico una lesión patológica resultante de la extravasación de moco a tejidos adyacentes llamados ránula, en la que presentó una respuesta clínica satisfactoria al tratamiento de la micromarsupalización modifica con hilo de algodón número 0 . La técnica de micromarsupalización modificada en el tratamiento de la ránula mostró éxito en el tratamiento, y tiene la ventaja de ser un procedimiento simple, tiempo operativo reducido, poco invasivo y con un menor riesgo de daño a las estructuras anatómicas.

Palabras clave: Ránula; Glándula Sublingual; Patología bucal.

\section{Introdução}

As glândulas salivares podem ser acometidas por vários processos patológicos, sejam eles de caráter inflamatório, alérgico, neoplásico, autoimune, cístico ou genético e a depender do tipo de afecção, a incidência será maior ou menor em um grupo determinado de glândulas salivares (Peterson, 2000). 
Rânula é caracterizada por um pseudocisto formado a partir da disseminação de muco nos tecidos moles gerado através de um trauma ou obstrução da glândula sublingual que geralmente ocorre de forma unilateral (Neville et al., 2016; Tommasi, 2013). Em relação ao acometimento sublingual dessas lesões e aspectos clínicos, as rânulas podem ser caracterizadas através de dois tipos: superficiais e mergulhantes ou cervicais (Moraes et al., 2015).

Clinicamente as rânulas superficiais são lesões que apresentam base séssil ou pediculada, de consistência firme ou flácida, superfície lisa e bem delimitada, medindo aproximadamente 1 centímetro de diâmetro. Geralmente possuem evolução lenta e assintomática, embora possa surgir de forma rápida e apresentar períodos de exacerbação e remissão, com sintomatologia dolorosa. Exibindo também uma coloração azulada pela transparência da rede capilar superficial e quando mais profundas nos tecidos são de cor semelhante à mucosa normal (Leal \& Braulio, 2014). As rânulas são vistas mais frequentemente em crianças e adultos jovens (Neville et al., 2016).

Uma variante clínica não usual, a rânula cervical ou mergulhante, ocorre quando a mucina extravasada disseca através do músculo milo-hióideo e produz o aumento de volume dentro do pescoço. Um aumento de volume concomitante no assoalho bucal pode ou não estar presente (Neville et al., 2016). Este tipo de lesão é caracterizada como rara e apresenta poucos casos na literatura (Moraes et al., 2015).

Embora o diagnóstico dessas lesões seja eminentemente clínico, faz-se necessário o estudo imagiológico complementar mais detalhado, com o auxílio de ultrassonografias, tomografias computadorizadas com contraste médio e, em casos de maior complexidade, é empregado o uso de ressonâncias magnéticas para efetuar o diagnóstico diferencial entre outras lesões de caráter císticas e neoplasias tumorais (Guzmán-Letelier et al., 2016).

Existem diversos métodos de tratamento que englobam marsupializações simples, marsupializações com uma gaze contendo iodo, excisão da lesão, excisão da rânula com a glândula sublingual, excisão a laser com vaporização da bolsa e tratamentos mais inovadores, com toxina botulínica tipo A e injeção intralesional de OK-432 (Oliveira et al., 2015).

\section{Metodologia}

Trata-se de um estudo caracterizado como relato de caso clínico de cunho qualitativo e descritivo acerca da utilização da técnica de micromarsupialização modificada no tratamento de rânula bilateral. Todos os princípios descritos por Helsinque em sua declaração foram respeitados, objetivando proteger a vida, saúde, privacidade e dignidade do ser humano. Além disso, o presente estudo apresenta o Termo de Consentimento Livre e Esclarecido (TCLE) para participação assinado pelo paciente, sendo relatadas todas as informações necessárias para a realização da documentação escrita e fotográfica do caso clínico, bem como a autorização para a publicação destas informações com objetivo científico.

\section{Relato de Caso Clínico}

Paciente A. M. A., 84 anos, sexo feminino, leucoderma, normocorada, hipertensa estágio 1, deu entrada no serviço de urgência/emergência em janeiro de 2019, com queixa de aumento de volume em região de assoalho bucal e discreta disfagia. Ainda na anamnese relatou que essa lesão passava por períodos de desenvolvimento e posterior remissão de forma espontânea, porém já estava há uma semana com lesão sem sinais de regressão.

Ao exame físico extra-oral, nenhuma alteração digna de nota. Na oroscopia, observou-se aumento de volume em região sublingual com acometimento bilateral, de coloração translúcida, flácida à palpação e indolor ao toque (Figura 1). 
Figura 1. Aspecto clínico intraoral.

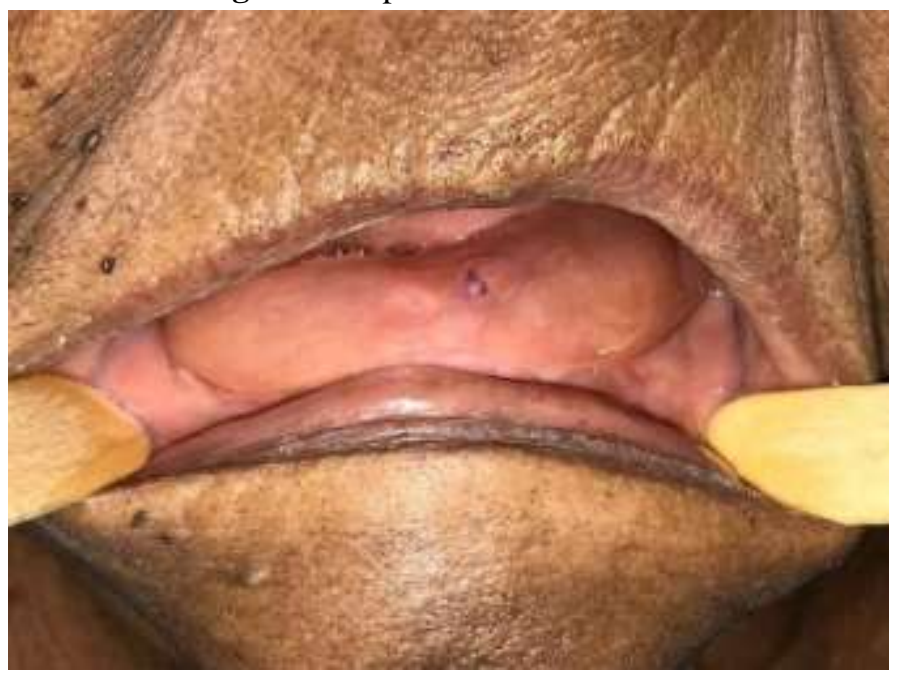

Fonte: Autores.

Solicitou-se uma radiografia oclusal inferior (Figura 2) e ultrassonografia das glândulas sublinguais (Figura 3) e submandibulares (Figura 4) para melhor avaliação e complementação de diagnóstico, onde observou-se a ausência de fragmentos radiopacos sugestivos a sialólitos e as referidas glândulas com dimensões e simetrias preservadas, sem evidências de formações císticas.

Figura 2. Radiografia oclusal inferior.

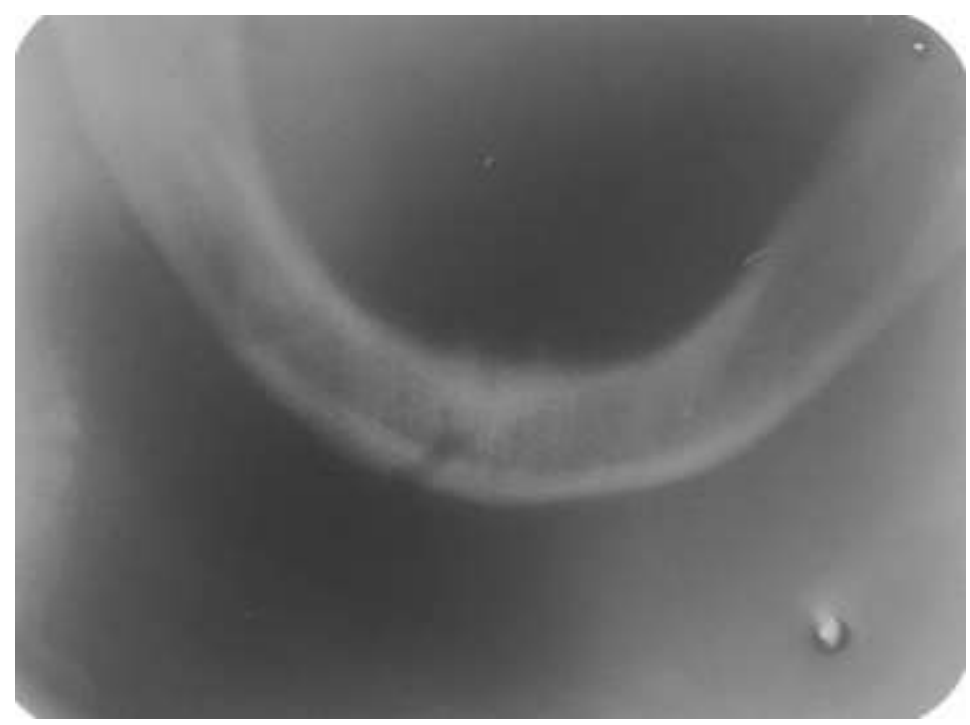

Fonte: Autores. 
Figura 3. Ultrassonografia da glândula sublingual.

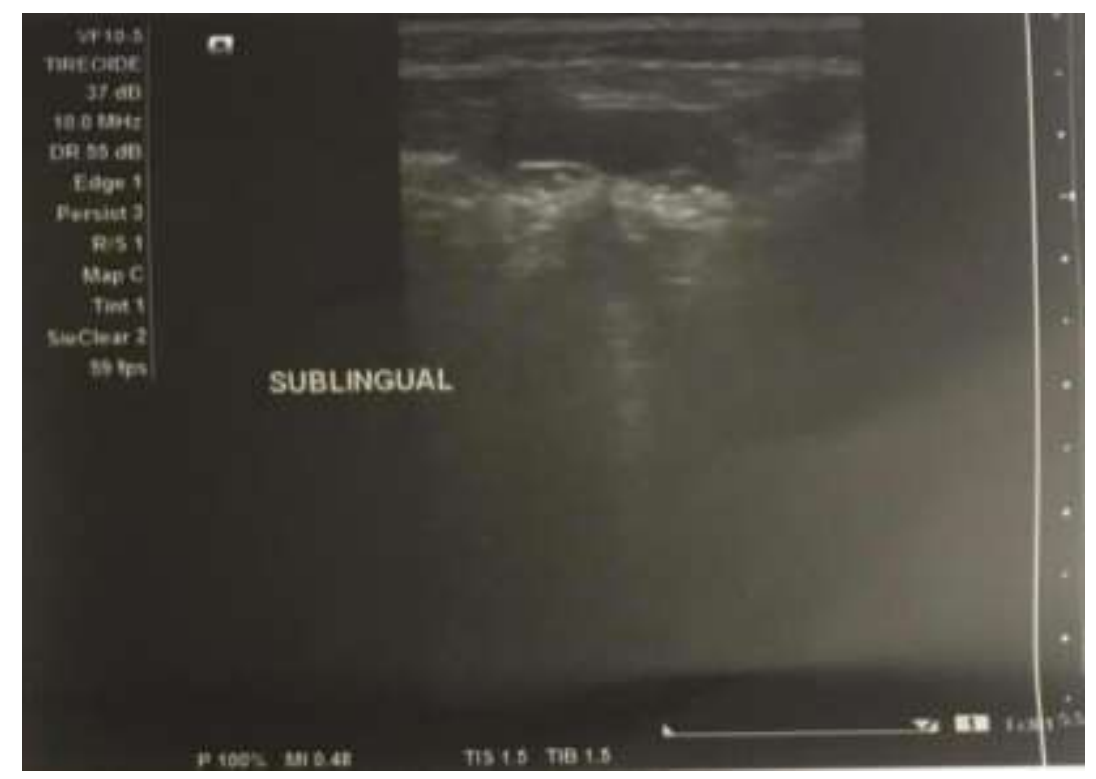

Fonte: Autores.

Figura 4. Ultrassonografia das glândulas submandibulares.

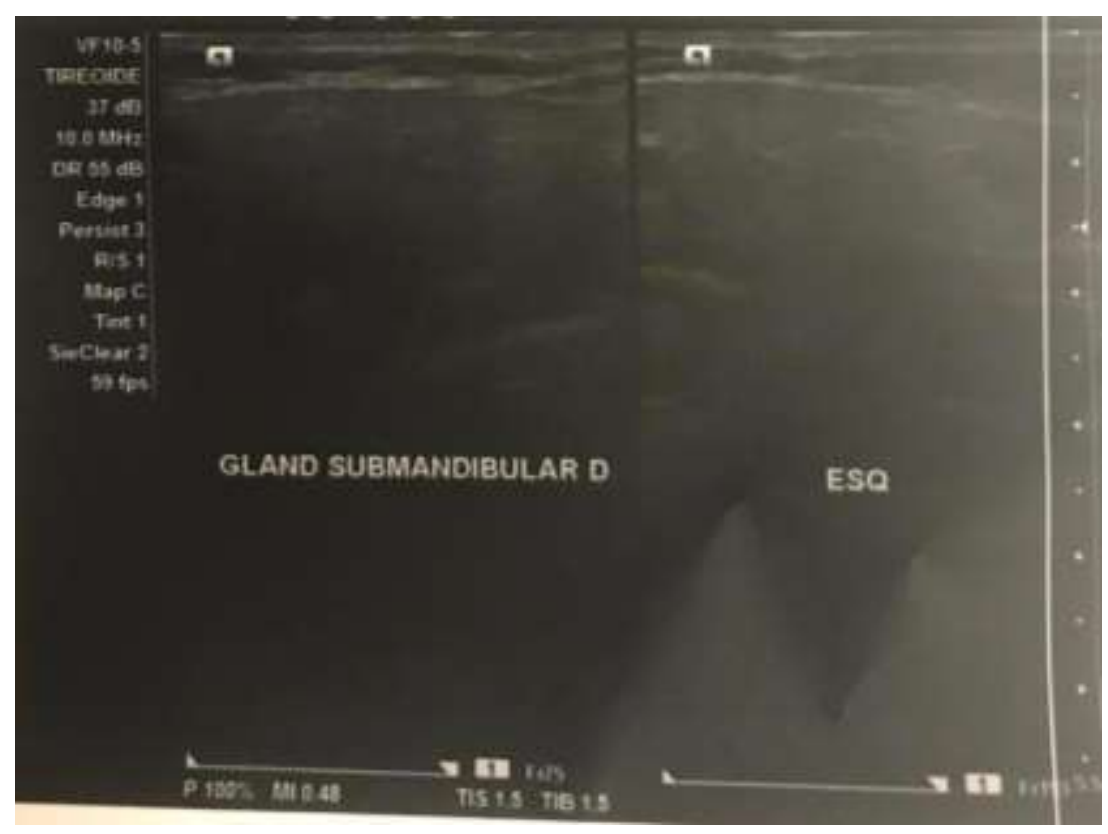

Fonte: Autores.

Frente ao diagnóstico, decidiu-se utilizar técnica de micromarsupialização modificada devido ao seu teor minimamente invasivo e traumático (Figura 4). Onde foram transfixadas 4 suturas com algodão 0 (Shalon medical), na superfície da lesão, apresentando uma redução significativa da distância entre os pontos de entrada e saída da agulha, permitindo assim a drenagem gradativa do conteúdo líquido no interior da lesão patológica. 
Figura 5. Técnica de micromarsupialização modificada.

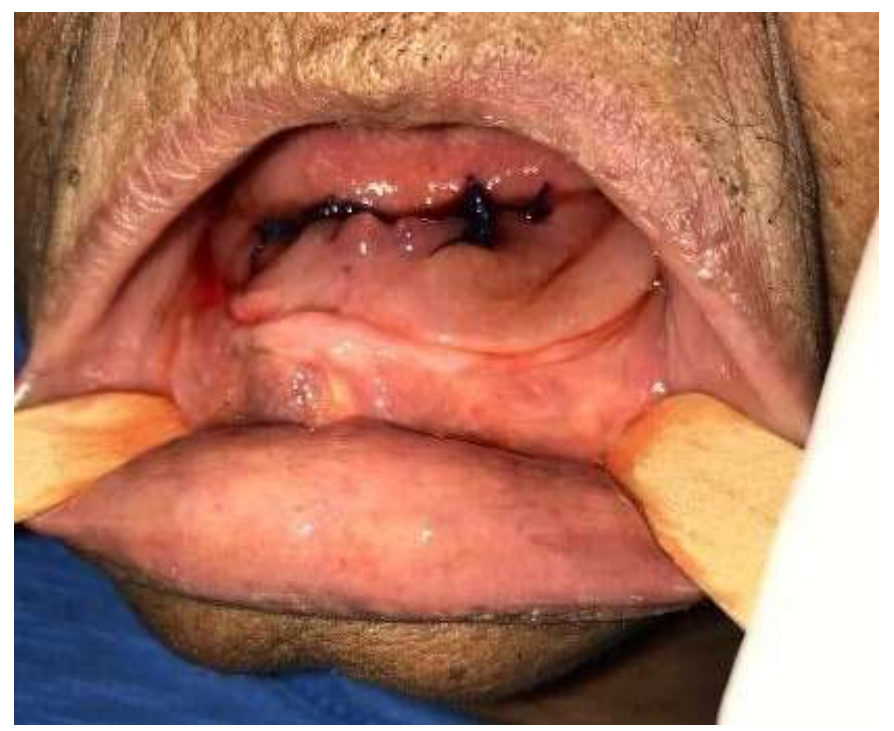

Fonte: Autores.

Realizou-se o acompanhamento de 1 ano e 11 meses (Figura 6) e a paciente segue sem apresentar sinais de recidiva da lesão.

Figura 6. Avaliação pós operatória de 1 ano e 11 meses.

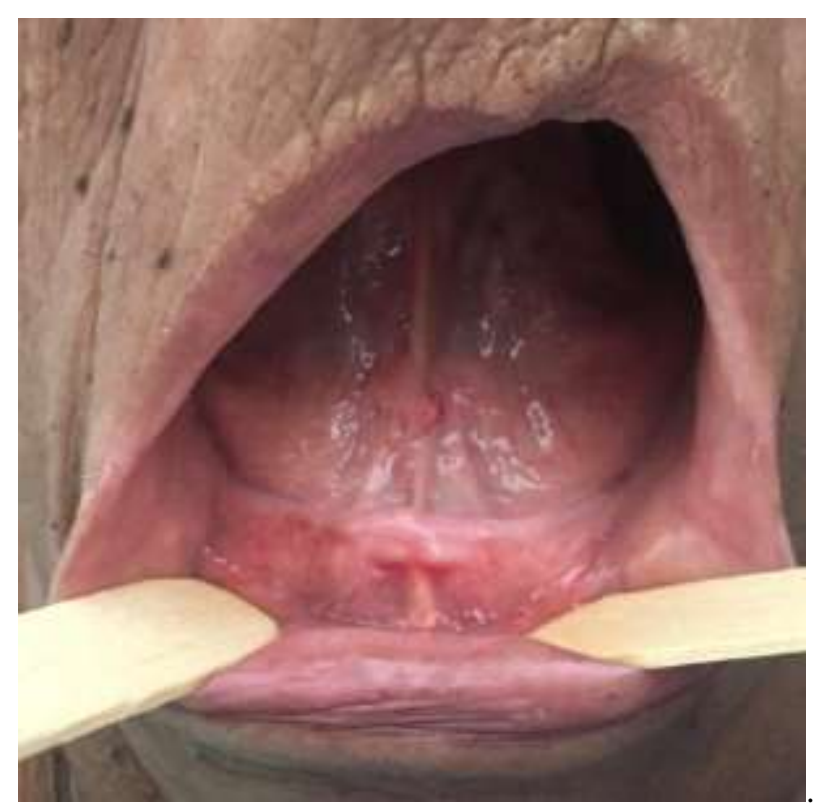

Fonte: Autores.

\section{Discussão}

Em geral, a rânula se apresenta como aumento de volume arredondado flutuante, de coloração azulada, no assoalho bucal, embora lesões profundas possam apresentar coloração normal da mucosa, seu tamanho costuma variar e a depender de sua localização e dimensão, pode interferir na fala e deglutição, causando certo desconforto (Alqhtani \& Krishnan, 2011; Guzmán-Letelier et al., 2016). 
As rânulas, na maioria dos casos, são localizadas unilateralmente (Lawal, et al., 2012). Em relação ao seu acometimento, são encontradas com maior frequência em pacientes pediátricos e adultos jovens (Neville et al., 2016; Kamalakaran et al., 2018; Matondkar, Yavagal \& Mandroli, 2019). Tal predominância talvez se dê pelo fato destes pacientes serem submetidos com maior frequência a traumatismos que induzem ao extravasamento de mucina (Noleto et al., 2010). No caso relatado a paciente apresenta 84 anos de idade e apresenta uma rânula bilateral, fugindo assim do padrão de localização e frequência de acometimento em relação a idade.

O diagnóstico é feito dado através da associação de características clínicas e pela punção com drenagem da saliva viscosa, faz-necessário também a análise de exames complementares imaginológicos como radiografias, ultrassonografias (USG), sialografia e tomografias computadorizadas para efetuar o diagnóstico diferencial dentre outras lesões que podem ocasionar obstrução dos ductos salivares por cálculos e tampões celulares (Freitas et al., 2004; Tomassi, 2013).

A USG é uma ferramenta de diagnóstico amplamente disponível, relativamente barato, não invasivo e facilmente reproduzível (Shah \& Asrani, 2017). Este exame complementar possibilita a distinção da lesão dos tecidos adjacentes superficiais localizados na região maxilofacial, sendo útil para fornecer informações sobre a natureza, tamanho e profundidade da lesão, bem como sua relação com vasos sanguíneos adjacentes e outras estruturas, além de ser um método altamente preciso no diagnóstico de neoplasias benignas e inchaços orofaciais causados por lesões císticas de tecidos moles ou inchaço inflamatório da região da cabeça e pescoço (de Castro et al., 2019). Por estes motivos, a USG foi eleita para o caso relatado como exame complementar de escolha.

Existem uma variedade de procedimentos cirúrgicos citados na literatura que varia de simples aspiração, excisão parcial ou completa da rânula e/ou da glândula salivar sublingual, às vezes envolvendo a glândula salivar submandibular. Podendo também lançar mão de tratamentos como: marsupialização, dissecção, crioterapia, escleroterapia, hidrodissecção e ablação a laser (Kokong et al., 2017).

O gerenciamento de rânulas é um tópico polarizador, com evidências conflitantes sobre qual a melhor modalidade de tratamento devido a lacuna existente no conhecimento sobre o conceito atual de sua etiopatogenia. O tratamento usual da rânula é a marsupialização e em casos de rânulas recorrentes ou persistentes o tratamento de escolha deve ser a excisão da rânula e da glândula sublingual (Peterson, 2000).

Apesar da maior taxa de recorrência da marsupialização, ainda assim é um método de tratamento cirúrgico muito utilizado, talvez por ser mais conservador e, portanto, diminuir o risco potencial de envolver estruturas anexas ou, até mesmo, com o intuito de tentar preservar a função da glândula sublingual (Oliveira et al., 2015).

A técnica de micromarsupialização foi descrita inicialmente por Cardoso em 1974. (Rocha et al., 2013). E, posteriormente, vários estudiosos efetuaram modificações visando a melhoria da técnica. A técnica de micromarsupialização modificada consiste na realização de suturas sucessivas com um distanciamento pequeno entre a saída e entrada da agulha com o objetivo de criar novos canalículos de drenagem com formação epitelial, onde devem permanecer durante 30 dias (Jiménez, Fernández \& Arce, 2016). O procedimento é simples, rápido, pouco traumático, bem tolerado pelo paciente, de baixo custo e, quando realizado de forma adequada, pode promover resultados satisfatórios.

O número de suturas realizadas é variável de acordo com o tamanho da lesão. A distância entre a entrada e a saída da agulha é importante e deve ser diminuída, a fim de reduzir o comprimento dos ductos de drenagem e facilitar a epitelização das novas vias formadas pelas suturas (Hegde, Bubna \& Rao, 2017).

O tempo para realizar o procedimento é rápido, levando cerca de 3 minutos, não provoca nenhum dano tecidual ou inflamação e é particularmente adequado para crianças pequenas que não toleram procedimentos longos ou invasivos (Negromonte Gonçalves et al., 2019). No caso relatado devido a idade avançada da paciente, promover a proteção de estruturas 
adjacentes relevantes e visando mitigar possíveis complicações pós-operatórias, a micromarsupialização foi a técnica de eleição.

\section{Conclusão}

A técnica da micromarsupialização no tratamento de rânula não mergulhante mostrou-se eficaz e apresenta inúmeras vantagens em relação a outros tipos de tratamento, por se tratar de um procedimento pouco invasivo, com menor chance de lesão a estruturas anatômicas nobres da região e reduzido tempo operatório.

\section{Referências}

Alqhtani, N. R., \& Krishnan, A. (2011). Ranula: pathogenesis and management-A review. IJD. International Journal of Dentistry, 11(1), 49-54.

de Castro, T. F., Quinto, J. H. S., Iwaki, L. C. V., Veltrini, V. C., Iwaki Filho, L., \& de Souza Tolentino, E. (2019). A ultrassonografia como ferramenta no diagnóstico do lipoma em região maxilofacial: relato de caso. Revista uningá, 56(S3), 11-17.

Freitas, S. E. N. D., Furuse, C. F., Biazolla, E. R., \& Andrade Sobrinho, J. (2004). Rânula: técnica de marsupialização: relato de caso clínico. Rev. Odontol. Araçatuba (Impr.), 53-56.

Guzmán-Letelier, M., Crisosto-Jara, C., Reyes-Marislao, B., Peñarrocha-Diago, M., \& Peñarrocha-Oltra, D. (2016). Marsupialización modificada de ránula gigante intraoral: reporte de caso. Revista clínica de periodoncia, implantología y rehabilitación oral, 9(3), $217-221$.

Hegde, S., Bubna, K., \& Rao, D. (2017). Management of ranula in a child by modified micro-marsupialization technique: a case report. Journal of Clinical Pediatric Dentistry, 41(4), 305-307.

Jiménez, D. R., Fernández, I. P., \& Arce, D. Z. (2017). Ránula, alternativas de tratamiento quirúrgico versus no quirúrgico. Odovtos-International Journal of Dental Sciences, 18(1E), 15-28.

Kamalakaran, A., Jayaraman, B., Balasubramaniam, S., Thirunavukkarasu, R., \& Ramakrishnan, B. (2018). Plunging ranula in a 78-year-old male-a rare case report. Journal of clinical and experimental dentistry, 10(1), e92.

Kokong, D., Iduh, A., Chukwu, I., Mugu, J., Nuhu, S., \& Augustine, S. (2017). Ranula: current concept of pathophysiologic basis and surgical management options. World Journal of Surgery, 41(6), 1476-1481.

Lawal, A. O., Adisa, A. O., Kolude, B., Adeyemi, B. F., \& Olajide, M. A. (2013). A review of 413 salivary gland tumours in the head and neck region. Journal of clinical and experimental dentistry, 5(5), e218.

Leal, R. M., \& Braulio, I. T. (2014). Marsupialização em rânula: relato de caso clínico. Arq Bras Odontol, 10(1), 15-20.

Matondkar, S. P., Yavagal, C., \& Mandroli, P. S. (2019). Modified micro-marsupialization as an alternative treatment for the management of ranulas in children. National journal of maxillofacial surgery, 10(1), 95.

Moraes, P. D. C., Teixeira, R. G., Thomaz, L. A., Junqueira, J. L. C., \& Oliveira, L. B. (2015). Cryosurgery for the treatment of pediatric plunging ranula: a conservative management. RGO-Revista Gaúcha de Odontologia, 63(4), 492-495.

Negromonte Gonçalves, K. K., dos Santos, M. S., de Ângelis Alves Silva, J., \& Lira Correia, A. V. (2019). Rânula mergulhante extensa em paciente pediátrico tratado com micromarsupialização: relato de caso. RSBO: Revista Sul-Brasileira de Odontologia, 16(2).

Neville, B. W., Damm, D. D., Allen, C. M., \& Chi, A. C. (2016). Patologia Oral e Maxilofacial (4a ed.), Elsevier.

Noleto, J. W., Israel, M., Mourão, C. F., \& de Sá Bonfim, T. (2010). Rânula mergulhante tratada por meio de marsupialização: relato de caso. Revista Brasileira de Odontologia, 67(1), 60 .

Oliveira, J. C. S. D., Garcia-Júnior, I. R., Camelo, J., Rabêlo, L. R. S., \& Bastos, E. G. (2015). Rânula mergulhante recidivante: relato de caso. Revista de Cirurgia e Traumatologia Buco-maxilo-facial, 15(2), 27-32.

Peterson, L. J. (2000). Cirurgia Oral e Maxilofacial Contemporânea (3a ed.). Guanabara Koogan.

Rocha, A. L., Basílio, J. M. B. S., Amaral, M. B. F., \& Mesquita, R. A. (2013). Tratamento da mucocele com a técnica da micromarsupialização modificada. Revista da Associação Paulista de Cirurgiões Dentistas, 67(4), 268-271. 
Research, Society and Development, v. 10, n. 4, e21610414032, 2021

(CC BY 4.0) | ISSN 2525-3409 | DOI: http://dx.doi.org/10.33448/rsd-v10i4.14032

Shah, J. S., \& Asrani, V. K. (2017). Clinical applications of ultrasonography in diagnosing head and neck swellings. Journal of Oral and Maxillofacial Radiology, 5(1), 7.

Tommasi, M. H. (2013). Diagnóstico em Patologia Bucal (4a ed.), Elsevier. 